prospective career pathway of the trainee". This experience must be appropriately managed, supervised and assessed.

Method. We conducted a survey of Speciality trainees in the West Midlands region across all psychiatric specialities using an online survey. The survey was open for one month period in January 2021 and reminders were sent intermittently. Following survey closure, quantitative data were analysed using Google Forms and Excel. Qualitative data were collated and reviewed to identify relevant themes.

Result. 47 of the total 82 Speciality trainees in all psychiatric specialities including dual trainees responded. Maximum response rate was from General adult/Dual trainees who form the bulk of Speciality trainees. Most trainees discussed their special interest with their supervisors and included this in learning plans. 79\% were able to have a weekly session. Most sessions were devoted to gaining additional clinical experience, medical education, gaining leadership competencies and completion of further post graduate qualifications. The majority of trainees chose special interest sessions in their own trust, however $45 \%$ had difficulty getting released from their clinical commitments. Trainees demonstrated evidence in their portfolio by reflection, WPBA and reflective notes. Trainees were positive about their experiences and requested more support to access sessions locally.

Conclusion. The Future Doctor report (HEE 2020) recognised that our Future Doctors must have a broad range of generalist skills to meet the population needs, therefore it is essential that doctors in training are supported by trainers and trusts to access special interest sessions to ensure that they achieve a broad range of competencies. To signpost trainees we have developed a booklet advertising available opportunities for ST trainees and other services may wish to consider this.

\section{Assessing wellbeing in foundation doctors during the COVID-19 pandemic}

Nikhita Handa* and Sanjeev Pramanik

East Lancashire Hospitals NHS Trust

${ }^{*}$ Corresponding author.

doi: 10.1192/bjo.2021.513

Aims. The COVID-19 pandemic has had a drastic effect on the mental health of the global population that is likely to be felt for years to come. One group particuarly likely to be affected by this in the immediate future are the healthcare professionals working on the frontline of the NHS pandemic response. As members of a foundation cohort of these junior doctors we aimed to create a way to quanitfy the wellbeing of ourselves and our colleagues at this challeging time. We aimed to use a combination of numerous tools to monitor foundation doctors in Blackburn during this crisis. This would inform which measures would be best suited to be put in place to protect this cohort from early burnout and poor mental health in the future.

Method. We designed a survey of 25 questions which we invited our foundation colleagues to fill in anonymously during the first and second waves of the pandemic in response to times when foundation doctors were redeployed to aid the frontline. The survey has been based on the PHQ9, GAD7, Epworth Sleepiness scale, Physician wellbeing index, Medical students wellbeing index, Maslach burnout inventory BMA burnout questionnaire and the QOL scale.

Result. From a cohort of around 140 foundation doctors we had 46 participants in our trial of this tool; $46 \%$ had been redeployed and $54 \%$ not redeployed. Over $50 \%$ of survey respondents reported high stress, poor motivation and depersonalisation over the two weeks at the peak of the pandemic, key early signs of burnout. Lack of interest in their work, poor sleep and anhedonia were increased across both groups (redeployed and non redeployed). The interventions after the first wave data which repondents found beneficial included; financial reassurances during redeployments, protected non clinical areas for rest, a named individual senior staff member for wellbeing support.

Conclusion. Key issues the survey raised were fed back to foundation programme leads in monthly meetings. This allowed us with our foundation leads to make targeted changes in order to support foundation doctors at this time. Without the data from this tool which we tailored to the foundation experience we believe these rapidly worsening issues during the pandemic would not have been addressed so swiftly. We then resurveyed the foundation cohort to assess which of these interventions have been most widely used and appreciated.

\section{Substance misuse teaching: a patient safety issue}

Mary Thornton ${ }^{1}$, Lucy Harborow ${ }^{1 \star}$, Emmert Roberts ${ }^{2}$, Nicola J. Kalk ${ }^{3}$ and Mike Kelleher ${ }^{4}$

${ }^{1}$ South London and Maudsley NHS Foundation Trust, Kings College London; ${ }^{2}$ South London and Maudsley NHS Foundation Trust, Kings College London, Institute of Psychiatry, Psychology and

Neuroscience, King's College London; ${ }^{3}$ South London and Maudsley NHS Foundation Trust, Kings College London, Kings College London and ${ }^{4}$ South London and Maudsley NHS Foundation Trust ${ }^{\star}$ Corresponding author.

doi: $10.1192 / b j o .2021 .514$

Aims. Clinical substance misuse presentations are commonly managed by Psychiatry Core Trainees (CTs) out of hours. However, specialist teaching is not included in the Maudsley Training Program (MTP) induction. We aimed to investigate whether this was of clinical concern and, if so identify interventions to address it.

Background. The association of substance misuse disorder and mental illness is widely recognised. The Adult Psychiatric Morbidity Survey 2014 reported that half of people dependent on drugs other than cannabis were receiving mental health treatment. Substance use substantially impacts clinical risk; 57\% of patient suicides in 2017 had a history of substance misuse. It also effects emergency psychiatric services: $55-80 \%$ of patients detained under S136 are intoxicated. Therefore, it is imperative for patient safety that CTs can assess and manage these patients appropriately.

The Royal College of Psychiatrists recognises the need for specialist substance misuse knowledge and skills, and lists this as a key 'Intended Learning Outcome' for CTs. Unfortunately, the availability of specialist drug and alcohol service placements for CTs has significantly declined. Only one placement is available per MTP rotation. Teaching is therefore relied upon to gain these competencies.

Method. Using a cross-sectional survey we explored CTs confidence in recognising and managing substance misuse presentations, knowledge of where to seek guidance and asked for teaching suggestions. We surveyed two CT1 cohorts in 2017 and 2019.

Result. Fifty-one CTs took the survey. Of these $92 \%$ did not feel prepared to manage acute substance intoxication or withdrawal and $96 \%$ would like relevant teaching at the start of CT1. Furthermore, $67 \%$ did not know where they could seek guidance.

CTs felt confident at recognising and managing alcohol related presentations. However, they were less confident in recognising opioid withdrawal, how to safely prescribe opioid substitution 